\title{
ON THE EXTENSION PROPERTY OF REIFENBERG-FLAT DOMAINS
}

\author{
Antoine Lemenant, Emmanouil Milakis and Laura V. Spinolo \\ Université Paris Diderot - Paris 7 - LJLL - CNRS, U.F.R. de Mathématiques \\ Bâtiment Sophie Germain, 75205 Paris Cedex 13, France; lemenant@ljll.univ-paris-diderot.fr \\ University of Cyprus, Department of Mathematics \& Statistics \\ P.O. Box 20537, Nicosia, CY-1678 Cyprus; emilakis@ucy.ac.cy
}

Istituto di Matematica Applicata e Tecnologie Informatiche, Consiglio Nazionale delle Ricerche via Ferrata 1, I-27100, Pavia, Italy; spinolo@imati.cnr.it

\begin{abstract}
We provide a detailed proof of the fact that any open set whose boundary is sufficiently flat in the sense of Reifenberg is also Jones-flat, and hence it admits an extension operator. We discuss various applications of this property, in particular we obtain $L^{\infty}$ estimates for the eigenfunctions of the Laplace operator with Neumann boundary conditions. We also compare different ways of measuring the "distance" between two Reifenberg-flat domains. These results are pivotal to the quantitative stability analysis of the spectrum of the Neumann Laplacian performed in [27].
\end{abstract}

\section{Introduction}

The main goal of the present paper is establishing extension and geometric properties for a class of open sets whose boundaries satisfy a fairly weak regularity requirement introduced by Reifenberg [29]. In particular, we show that any open set whose boundary is sufficiently flat in the sense of Reifenberg enjoys the so-called extension property and we discuss applications that are relevant for the analysis of PDEs defined in these sets. We also compare different ways of measuring the "distance" between two sufficiently close Reifenberg-flat domains $X$ and $Y$, in particular we discuss the relations between the Hausdorff distances $d_{H}(X, Y), d_{H}\left(\mathbf{R}^{N} \backslash X, \mathbf{R}^{N} \backslash Y\right)$ and $d_{H}(\partial X, \partial Y)$ and the measure of the symmetric difference $|X \triangle Y|$.

Although we are confident our results can find different applications, our original motivation was the quantitative stability analysis of the spectrum of the Laplace operator with Neumann boundary conditions defined in Reifenberg-flat domains, see [27].

The notion of Reifenberg-flat set was first introduced in 1960 by Reifenberg [29] when he was working on the Plateau problem, and has since then played an important role in the study of minimal surfaces. More recently, the works by David [10, 11] about the regularity for 2-dimensional minimal sets in $\mathbf{R}^{N}$ rely on the Reifenberg parametrization and the specific 3-dimensional results by David, De Pauw and Toro [13]. Also, Reifenberg-flat set are relevant in the study of the harmonic measure (see Kenig and Toro [20, 21, 22] and Toro [32, 33]) and of the regularity for free boundary problems, like the minimization of the Mumford-Shah functional (see [23,

doi:10.5186/aasfm.2014.3907

2010 Mathematics Subject Classification: Primary 49Q20, 49Q05, 46E35.

Key words: Reifenberg-flat sets, extension operators. 
24]). A Reifenberg-flat domain is known in the literature as being an open set which admits a Reifenberg-flat set as boundary. Elliptic and parabolic equations defined in Reifenberg-flat domains have been recently investigated by Byun, Wang and Zhou [2, 3, 4], by Lemenant, Milakis and Spinolo and by Milakis and Toro [25, 26, 27, 28]. Finally, we mention that Reifenberg-flat domains are in particular NTA domains in the sense of Jerison and Kenig [18].

We now provide the precise definition. We denote by $d_{H}$ the classical Hausdorff distance between two sets $X$ and $Y$,

$$
d_{H}(X, Y):=\max \left\{\sup _{x \in X} d(x, Y), \sup _{y \in Y} d(y, X)\right\} .
$$

Definition 1. Let $\varepsilon, r_{0}$ be two real numbers satisfying $0<\varepsilon<1 / 2$ and $r_{0}>0$. A nonempty open set $\Omega \subseteq \mathbf{R}^{N}$ is a $\left(\varepsilon, r_{0}\right)$-Reifenberg-flat-domain if it satisfies the following two conditions:

i) for every $x \in \partial \Omega$ and for every $r \leq r_{0}$, there is a hyperplane $P(x, r)$ containing $x$ which satisfies

$$
\frac{1}{r} d_{H}(\partial \Omega \cap B(x, r), P(x, r) \cap B(x, r)) \leq \varepsilon .
$$

ii) For every $x \in \partial \Omega$, one of the connected component of

$$
B\left(x, r_{0}\right) \cap\left\{y: \operatorname{dist}\left(y, P\left(x, r_{0}\right)\right) \geq 2 \varepsilon r_{0}\right\}
$$

is contained in $\Omega$ and the other one is contained in $\mathbf{R}^{N} \backslash \Omega$.

Remark 2. According to our definition, a Reifenberg-flat-domain is not necessarily a domain, in the sense that it is not necessarily connected. We prefer to still use the terminology "domain" to be consistent with the literature and avoid confusion with the definition of Reifenberg-flat-set, which is satisfied by the boundary of a Reifenberg flat domain. Throughout this paper we will sometimes say that an open set is Reifengerg-flat, which means that it is a Reifenberg-flat-domain.

A Reifenberg-flat-domain enjoys local separability properties (see e.g. Theorem 4.1 in [16]), however we observe that condition ii) in the definition is not in general implied by condition i), as the example of $\Omega=\mathbf{R}^{N} \backslash \partial B(0,1)$ shows (here $\partial B(0,1)$ denotes the boundary of the unit ball). However, a consequence of the analysis in David [12] is that i) implies ii) under some further topological assumption, for instance the implication holds if $\Omega$ and $\partial \Omega$ are both connected. Note furthermore that a straightforward consequence of the definition is that, if $\varepsilon_{1}<\varepsilon_{2}$, then any $\left(\varepsilon_{1}, r_{0}\right)$-Reifenberg-flat-domain is also $\left(\varepsilon_{2}, r_{0}\right)$-Reifenberg-flat. Finally, note that we only impose the separability requirement ii) at scale $r_{0}$ but it simply follows from the definition that it also holds at any scale $r \leq r_{0}$ (see [20, Proposition 2.2] or Lemma 6 below).

In [29] Reifenberg proved the so-called Topological Disk Theorem which states that, provided $\varepsilon$ is small enough, the boundary of any $\left(\varepsilon, r_{0}\right)$-Reifenberg-flat open set in the unit $N$-ball is the bi-Hölder image of an $(N-1)$-dimensional disk. Also, any Lipschitz open set with sufficiently small Lipschitz constant is Reifenberg-flat for a suitable choice of the regularity parameter $\varepsilon$ (the choice depends on the Lipschitz constant). On the other hand, the "flat" Koch snowflake with sufficiently small angle is Reifenberg-flat (see Toro [32]) and hence it is an example of a Reifenberg-flat set which is not Lipschitz, and with Hausdorff dimension greater than $N-1$. 
The main goal of this paper is providing a complete and detailed proof of the fact that Reifenberg-flat-domains admit extension operators. This fact is relevant for the study of elliptic problems and was already known and used in the literature (see e.g. the introduction of [2]). However, to the best of our knowledge, an explicit proof was so far missing. We recall that the so called extension problem can be formulated as follows: given an open set $\Omega$, we denote by $W^{1, p}$ the classical Sobolev space and we wonder whether or not one can define a bounded linear operator (the so-called extension operator)

$$
E: W^{1, p}(\Omega) \rightarrow W^{1, p}\left(\mathbf{R}^{N}\right)
$$

such that $E(u) \equiv u$ on $\Omega$. If $\partial \Omega$ is Lipschitz, Calderón [5] established the existence of an extension operator in the case when $1<p<\infty$, while Stein [31] considered the cases $p=1, \infty$. Jones [19] proved the existence of extension operators for a new class of so-called $\left(\delta, R_{0}\right)$-Jones flat sets (the precise definition is recalled in Section 2 ). In the present work we prove that open sets that are sufficiently flat in the sense of Reifenberg are indeed Jones flat. Our main result concerning the extension problem is as follows.

Theorem 3. Any $\left(1 / 600, r_{0}\right)$-Reifenberg-flat-domain is $\left(1 / 450, r_{0} / 7\right)$-Jones flat.

As a direct consequence of Theorem 3 we get that one can define extension operators for $\left(1 / 600, r_{0}\right)$-Reifenberg-flat-domains (see Corollary 9 for a precise statement). Some relevant features of this result are the following: first, we provide an explicit and universal threshold on the coefficient $\varepsilon$ for the extension property to hold (namely, $\varepsilon \leq 1 / 600$ ). Second, $1 / 600$ is fairly big compared to the usual threshold needed to apply Reifenberg Topological Disk Theorem (for e.g. the threshold is $10^{-15}$ in [13], see also [17] for an interesting alternative proof). Finally, note that we have used the threshold $\varepsilon=1 / 600$ to simplify some computations, but by straightforwardly following the proof of Theorem 3 one should get a slightly better threshold.

As a consequence of the extension property, we obtain that the classical RellichKondrachov Theorem applies to Reifenberg-flat-domains (see Proposition 11), that the Neumann Laplacian has a discrete spectrum and that the eigenfunctions are bounded (see Proposition 12). Also, by combining Theorem 3 with the works by Chua [7, 8, 9] and Christ [6] we get that one can define extension operators for weighted Sobolev spaces and Sobolev spaces of fractional order (see Remark 10 in the present paper).

We conclude the paper by establishing results unrelated to the extension problem, namely we study the relation between different ways of measuring the "distance" between sets of $\mathbf{R}^{N}$. In particular, for two general open sets $X$ and $Y$, neither the Hausdorff distance $d_{H}(X, Y)$ nor the Hausdorff distance between the complements $d_{H}\left(\mathbf{R}^{N} \backslash X, \mathbf{R}^{N} \backslash Y\right)$ is, in general, controlled by the Lebesgue measure of the symmetric difference $|X \triangle Y|$. However, we prove that they are indeed controlled provided that $X, Y$ are Reifenberg flat and close enough, in a suitable sense. This result will be as well applied in [27] to the stability analysis of the spectrum of the Laplace operator with Neumann boundary conditions. More precisely, Theorem 5 in [27] loosely speaking states that, if $X$ and $Y$ are two bounded open sets that are sufficiently flat in the sense of Reifenberg and sufficiently close in a suitable Hausdorff sense, then the following holds. The difference $\left|\mu_{n}(X)-\mu_{n}(Y)\right|$ is controlled in terms of $d_{H}(X, Y)$ and $d_{H}\left(\mathbf{R}^{N} \backslash X, \mathbf{R}^{N} \backslash Y\right)$. Here $\mu_{n}(X)$ and $\mu_{n}(Y)$ denote the $n$-th eigenvalues of the 
Laplace operator with homogeneous Neumann boundary conditions defined in $X$ and in $Y$, respectively. See [27, pp. 2102-2103] for the precise statement and for related comments.

The paper is organized as follows: in Section 2 we prove that open sets that are sufficiently flat in the sense of Reifenberg are Jones-flat, in Section 3 we show that if these sets are also connected, then they enjoy the extension property. In Section 3 we also discuss some applications of the extension property. In Section 4 we investigate how to handle sets that are not connected and finally in Section 5 we investigate the relation between different ways of measuring the "distance" between Reifenberg-flat-domains.

1.1. Notations. We denote by $C\left(a_{1}, \ldots, a_{h}\right)$ a constant only depending on the variables $a_{1}, \ldots, a_{h}$. Its precise value can vary from line to line. Also, we use the following notations:

$\mathcal{H}^{m}$ : the $m$-dimensional Hausdorff measure.

$\omega_{N}$ : the Lebesgue measure of the unit ball in $\mathbf{R}^{N}$.

$|A|$ : the Lebesgue measure of the Borel set $A \subseteq \mathbf{R}^{N}$.

$A^{c}$ : the complement of the set $A, A^{c}:=\mathbf{R}^{N} \backslash A$.

$\bar{A}$ : the closure of the set $A$.

$W^{1, p}(\Omega)$ : the Sobolev space of $L^{p}$ functions whose derivatives are in $L^{p}$.

$\langle x, y\rangle$ : the standard scalar product between the vectors $x, y \in \mathbf{R}^{N}$.

$|x|$ : the norm of the vector $x \in \mathbf{R}^{N}$.

$d(x, y)$ : the distance from the point $x$ to the point $y, d(x, y)=|x-y|$.

$d(x, A)$ : the distance from the point $x$ to the set $A$.

$d_{H}(A, B)$ : the Hausdorff distance from the set $A$ to the set $B$.

$[x, y]$ : the segment joining the points $x, y \in \mathbf{R}^{N}$.

$B(x, r)$ : the open ball of radius $r$ centered at $x$.

$\bar{B}(x, r)$ : the closed ball of radius $r$ centered at $x$.

\section{Reifenberg-flatness and Jones-flatness}

In this section we show that any open set that is sufficiently flat in the sense of Reifenberg is Jones-flat, in the sense of [19]. The extension property follows then as a corollary of the analysis in [19].

First, we provide the precise definition of Jones-flatness.

Definition 4. Given $\delta>0$ and $R_{0}>0$, an open set $\Omega$ is $\left(\delta, R_{0}\right)$-Jones-flat if for any $x, y \in \Omega$ such that $d(x, y) \leq R_{0}$ there is a rectifiable curve $\gamma \subseteq \Omega$ which connects $x$ and $y$ and satisfies

$$
\mathcal{H}^{1}(\gamma) \leq \delta^{-1} d(x, y)
$$

and

$$
d\left(z, \Omega^{c}\right) \geq \delta \frac{d(z, x) d(z, y)}{d(x, y)}, \quad \text { for all } z \in \gamma
$$

To investigate the relation between Jones flatness and Reifenberg flatness we need two preliminary lemmas.

Lemma 5. Let $\Omega \subseteq \mathbf{R}^{N}$ be an $\left(\varepsilon, r_{0}\right)$-Reifenberg-flat-domain. Given $x \in \partial \Omega$ and $r \leq r_{0}$, we term $\nu_{r}$ the unit normal vector to the hyperplane $P(x, r)$ provided by 
the definition of Reifenberg-flatness. Given $M \geq 1$, for every $r \leq r_{0} / M$ we have

$$
\left|\left\langle\nu_{r}, \nu_{M r}\right\rangle\right| \geq 1-(M+1) \varepsilon .
$$

Proof. We assume with no loss of generality that $x$ is the origin. For simplicity, in the proof we denote by $B_{r}$ the ball $B(0, r)$ and by $P_{r}$ the hyperplane $P(0, r)$. From the definition of Reifenberg flatness we infer that

$$
\begin{aligned}
d_{H}\left(P_{M r} \cap B_{r}, P_{r} \cap B_{r}\right) & \leq d_{H}\left(P_{M r} \cap B_{r}, \partial \Omega \cap B_{r}\right)+d_{H}\left(\partial \Omega \cap B_{r}, P_{r} \cap B_{r}\right) \\
& \leq M r \varepsilon+r \varepsilon \leq(M+1) r \varepsilon .
\end{aligned}
$$

Since $P_{M r}$ and $P_{r}$ are linear spaces we deduce that

$$
d_{H}\left(P_{M r} \cap B_{1}, P_{r} \cap B_{1}\right) \leq(M+1) \varepsilon .
$$

We term $\pi_{r}$ and $\pi_{M r}$ the orthogonal projections onto $P_{r}$ and $P_{M r}$, respectively, and we fix an arbitrary point $y \in P_{r} \cap B_{1}$. Inequality (2.4) states that there is $z \in \bar{P}_{M r} \cap \bar{B}_{1}$ satisfying

$$
d(z, y) \leq(M+1) \varepsilon
$$

In particular, since $1=\left|\nu_{M r}\right|=\inf _{z \in P_{M r}} d\left(\nu_{M r}, z\right)$, we get

$$
d\left(\nu_{M r}, y\right) \geq d\left(\nu_{M r}, z\right)-d(z, y) \geq 1-(M+1) \varepsilon .
$$

By taking the infimum for $y \in P_{r} \cap B_{1}$ we obtain

$$
\left|\nu_{M r}-\pi_{r}\left(\nu_{M r}\right)\right| \geq 1-(M+1) \varepsilon,
$$

and the proof is concluded by recalling that $\left|\left\langle\nu_{M r}, \nu_{r}\right\rangle\right|=d\left(\nu_{M r}, \pi_{r}\left(\nu_{M r}\right)\right)$.

The following lemma discusses an observation due to Kenig and Toro [20, Proposition 2.2]. Note that the difference between Lemma 6 and part ii) in the definition of Reifenberg flatness is that in ii) we only require the separation property at scale $r_{0}$.

Lemma 6. Let $\Omega \subseteq \mathbf{R}^{N}$ be an $\left(\varepsilon, r_{0}\right)$-Reifenberg-flat-domain. For every $x \in \partial \Omega$ and $\left.r \in] 0, r_{0}\right]$, one of the connected components of

$$
B(x, r) \cap\{y: \operatorname{dist}(y, P(x, r)) \geq 2 \varepsilon r\}
$$

is contained in $\Omega$ and the other one is contained in $\mathbf{R}^{N} \backslash \Omega$. Here $P(x, r)$ is the same hyperplane as in part i) of the definition of Reifenberg-flatness.

Proof. We fix $\left.\rho \in] 0, r_{0}\right]$ and we assume that the separation property holds at scale $\rho$, namely that one of the connected components of

$$
B(x, \rho) \cap\{y: \operatorname{dist}(y, P(x, \rho)) \geq 2 \varepsilon \rho\}
$$

is contained in $\Omega$ and the other one is contained in $\mathbf{R}^{N} \backslash \Omega$. We now show that the same separation property holds at scale $r$ for every $r \in] \rho / M, \rho]$ provided that $M \leq(1-\varepsilon) / 3 \varepsilon$. By iteration this implies that the separation property holds at any scale $\left.r \in] 0, r_{0}\right]$.

Let us fix $r \in] \rho / M, \rho]$ and denote by $B^{+}(x, r)$ one of the connected components of

$$
B(x, r) \cap\{y: \operatorname{dist}(y, P(x, r)) \geq 2 \varepsilon r\}
$$

and by $B^{-}(x, r)$ the other one. Also, we term $Y^{+}$and $Y^{-}$the points of intersection of the line passing through $x$ and perpendicular to $P(x, r)$ with the boundary of the ball $B(x, r)$. 
By recalling (2.3) and the inequality $r \geq \rho / M$, we get that the distance of $Y^{ \pm}$ from the hyperpane $P(x, \rho)$ satisfies the following inequality:

$$
d\left(Y^{ \pm}, P(x, \rho)\right) \geq r\left|\left\langle\nu_{\rho}, \nu_{\rho / M}\right\rangle\right| \geq r[1-(M+1) \varepsilon] \geq \rho \frac{1-(M+1) \varepsilon}{M} .
$$

Since by assumption $M \leq(1-\varepsilon) / 3 \varepsilon$, this implies that $d\left(Y^{ \pm}, P(x, \rho)\right) \geq 2 \varepsilon \rho$ and hence that one among $Y^{+}$and $Y^{-}$belongs to $B^{+}(x, \rho)$ and the other one to $B^{-}(x, \rho)$. Since by assumption the separation property holds at scale $\rho$, this implies that one of them belongs to $\Omega$ and the other one to $\Omega^{c}$.



Figure 1. Notations for the proof of Theorem 3.

To conclude, note that part i) in the definition of Reifenberg flatness implies that

$$
B^{ \pm}(x, r) \cap \partial \Omega=\emptyset
$$

and hence both $B^{+}(x, r)$ and $B^{-}(x, r)$ are entirely contained in either $\Omega$ or $\Omega^{c}$. By recalling that one among $Y^{+}$and $Y^{-}$belongs to $\Omega$ and the other one to $\Omega^{c}$, we conclude the proof of the lemma.

We are now ready to establish the main result of this section, namely Theorem 3.

Proof of Theorem 3. We assume $\varepsilon \leq 1 / 600$, we fix an $\left(\varepsilon, r_{0}\right)$-Reifenberg-flatdomain $\Omega \subseteq \mathbf{R}^{N}$ and we proceed according to the following steps.

$\diamond$ Step 1 . We first introduce some notations (see Figure 1 for a representation). For any $x_{0} \in \partial \Omega$ and $\rho \leq r_{0}$, we denote as usual by $P\left(x_{0}, \rho\right)$ the hyperplane provided by the definition of Reifenberg flatness, and by $\vec{\nu}_{\rho}$ its normal. By Lemma 6 , we can choose the orientation of $\vec{\nu}_{\rho}$ in such a way that

$$
B^{+}\left(x_{0}, \rho\right):=\left\{z+t \vec{\nu}_{\rho}: z \in P\left(x_{0}, \rho\right), t \geq 2 \varepsilon \rho\right\} \cap B\left(x_{0}, \rho\right) \subseteq \Omega
$$

and

$$
B^{-}\left(x_{0}, \rho\right):=\left\{z-t \vec{\nu}_{\rho}: z \in P\left(x_{0}, \rho\right), t \geq 2 \varepsilon \rho\right\} \cap B\left(x_{0}, \rho\right) \subseteq \Omega^{c} .
$$


Also, we define the hyperplanes $P^{+}\left(x_{0}, \rho\right)$ and $P^{-}\left(x_{0}, \rho\right)$ by setting

$$
P^{+}\left(x_{0}, \rho\right):=\left\{z+2 \varepsilon \rho \vec{\nu}_{\rho}: z \in P\left(x_{0}, \rho\right)\right\}
$$

and

$$
P^{-}\left(x_{0}, \rho\right):=\left\{z-2 \varepsilon \rho \vec{\nu}_{\rho}: z \in P\left(x_{0}, \rho\right)\right\}
$$

and we denote by $Y\left(x_{0}, \rho\right)$ the point

$$
Y\left(x_{0}, \rho\right):=x_{0}+\rho \vec{\nu}_{\rho} .
$$

Finally, for any $x \in \Omega$, we denote by $x_{0} \in \partial \Omega$ the point such that $d\left(x, \Omega^{c}\right)=$ $d\left(x, x_{0}\right)$ (if there is more than one such $x_{0}$, we arbitrarily fix one).

$\diamond$ Step 2. We provide a preliminary construction: more precisely, given

- $x \in \Omega$ such that $d\left(x, \Omega^{c}\right) \leq 2 r_{0} / 7$, and

- $r$ satisfying $d\left(x, \Omega^{c}\right) / 2 \leq r \leq r_{0} / 7$,

the curve $\gamma_{x, r}$ is defined as follows.

(I) If $d\left(x, \Omega^{c}\right) / 2 \leq r \leq 2 d\left(x, \Omega^{c}\right)$, then $\gamma_{x, r}$ is simply the segment $\left[x, Y\left(x_{0}, r\right)\right]$.

(II) If $2 d\left(x, \Omega^{c}\right)<r \leq r_{0} / 7$, we denote by $k_{0} \geq 1$ the biggest natural number $k$ satisfying $2^{-k} r \geq d\left(x, \Omega^{c}\right)$ and we set

$$
\gamma_{x, r}:=\left[x, Y\left(x_{0}, 2^{-k_{0}} r\right)\right] \cup \bigcup_{k=0}^{k_{0}-1}\left[Y\left(x_{0}, 2^{-k} r\right), Y\left(x_{0}, 2^{-(k+1)} r\right)\right] .
$$

$\diamond$ Step 3. We prove that in both cases (I) and (II) we have

$$
\mathcal{H}^{1}\left(\gamma_{x, r}\right) \leq 4 r .
$$

To handle case (I) we just observe that, since by assumption $d\left(x, \Omega^{c}\right)=d\left(x, x_{0}\right) \leq 2 r$, then, by recalling $d\left(x_{0}, Y\left(x_{0}, r\right)\right)=r$, property (2.5) follows.

To handle case (II), we first observe that, since $d\left(x, x_{0}\right) \leq 2^{-k_{0}} r$, then both $x$ and $Y\left(x_{0}, 2^{-k_{0}} r\right)$ belong to the closure of $B\left(x_{0}, 2^{-k_{0}} r\right)$. Also, by construction both $Y\left(x_{0}, 2^{-k} r\right)$ and $Y\left(x_{0}, 2^{-(k+1)} r\right)$ belong to the closure of $B\left(x_{0}, 2^{-k} r\right)$ and by combining these observations we conclude that

$$
\begin{aligned}
\mathcal{H}^{1}\left(\gamma_{x, r}\right) & =d\left(x, Y\left(x_{0}, 2^{-k_{0}} r\right)\right)+\sum_{k=0}^{k_{0}-1} d\left(Y\left(x_{0}, 2^{-k} r\right), Y\left(x_{0}, 2^{-(k+1)} r\right)\right) \\
& \leq 2 \cdot 2^{-k_{0}} r+\sum_{k=0}^{k_{0}-1} 2 \cdot 2^{-k} r \leq 2 r \sum_{k \in \mathbf{N}} 2^{-k}=4 r
\end{aligned}
$$

$\diamond$ Step 4. We prove that for every $z \in \gamma_{x, r}$

$$
d\left(z, \Omega^{c}\right) \geq \frac{29}{240} d(z, x) .
$$

We start by handling case (I): we work in the ball $B\left(x_{0}, 4 r\right)$ and we recall the definition of $B^{+}\left(x_{0}, 4 r\right)$ and of $B^{-}\left(x_{0}, 4 r\right)$, given at Step 1 . Since by assumption $\varepsilon \leq 1 / 32$, we have

$$
16 \varepsilon r \leq \frac{r}{2} \leq d\left(x, \Omega^{c}\right) \leq d\left(x, B^{-}\left(x_{0}, 4 r\right)\right)
$$

and hence $x \in B^{+}\left(x_{0}, 4 r\right)$. Let $\beta$ denotes the angle between $\nu_{r}$ and $\nu_{4 r}$, then by Lemma 5 applied with $M=4$ we get that provided $\varepsilon \leq 1 / 9$, then $4 \varepsilon r \leq r \cos \beta$, so that $Y\left(x_{0}, r\right) \in B^{+}\left(x_{0}, 4 r\right)$. By recalling that $x \in B^{+}\left(x_{0}, 4 r\right)$, we conclude that $\left[x, Y\left(x_{0}, r\right)\right] \subseteq B^{+}\left(x_{0}, 4 r\right)$. 
We are now ready to establish (2.7), so we fix $z \in\left[x, Y\left(x_{0}, r\right)\right]$. To provide a bound from above on $d(z, x)$, we simply observe that, since both $x$ and $Y\left(x_{0}, r\right)$ belong to the closure of $B\left(x_{0}, 2 r\right)$, then so does $z$ and hence

$$
d(z, x) \leq 4 r .
$$

Next, we provide a bound from below on $d\left(z, \Omega^{c}\right)$ : since $z \in B^{+}\left(x_{0}, 4 r\right) \subseteq \Omega$, then

$$
d\left(z, \Omega^{c}\right) \geq d\left(z, \partial B^{+}\left(x_{0}, 4 r\right)\right)=\min \left\{d\left(z, P^{+}\left(x_{0}, 4 r\right)\right), d\left(z, \partial B\left(x_{0}, 4 r\right)\right)\right\} .
$$

First, we recall that $z \in B\left(x_{0}, 2 r\right)$ and we provide a bound on the distance from $z$ to the spherical part of $\partial B^{+}\left(x_{0}, 4 r\right)$ :

$$
d\left(z, \partial B\left(x_{0}, 4 r\right)\right)=4 r-d\left(z, x_{0}\right) \geq 4 r-2 r=2 r .
$$

Next, we observe that

$$
d\left(z, P^{+}\left(x_{0}, 4 r\right)\right)=d\left(z, P\left(x_{0}, 4 r\right)\right)-8 \varepsilon r
$$

and, since $z \in\left[x, Y\left(x_{0}, r\right)\right]$, then

$$
d\left(z, P\left(x_{0}, 4 r\right)\right) \geq \min \left\{d\left(x, P\left(x_{0}, 4 r\right)\right), d\left(Y\left(x_{0}, r\right), P\left(x_{0}, 4 r\right)\right)\right\} .
$$

Note that $d\left(Y\left(x_{0}, r\right), P\left(x_{0}, 4 r\right)\right)=r \cos \beta$ and, using Lemma 5 , we conclude that

$$
d\left(Y\left(x_{0}, r\right), P\left(x_{0}, 4 r\right)\right) \geq r / 2
$$

because $\varepsilon \leq 1 / 10$. Also, since $B^{-}\left(x_{0}, 4 r\right) \subseteq \Omega^{c}$, then

$$
r / 2 \leq d\left(x, \Omega^{c}\right) \leq d\left(x, B^{-}\left(x_{0}, 4 r\right)\right)=d\left(x, P\left(x_{0}, 4 r\right)\right)+2 \varepsilon r .
$$

By recalling (2.9) and the inequality $\varepsilon \leq 1 / 600$ and by combining all the previous observations we conclude that

$$
\begin{aligned}
d\left(z, \Omega^{c}\right) & \geq d\left(z, \partial B^{+}\left(x_{0}, 4 r\right)\right) \geq \min \left\{d\left(z, P^{+}\left(x_{0}, 4 r\right)\right), 2 r\right\} \\
& =\min \left\{d\left(z, P\left(x_{0}, 4 r\right)\right)-8 \varepsilon r, 2 r\right\} \geq \\
& \geq \min \left\{\min \left\{d\left(x, P\left(x_{0}, 4 r\right)\right), d\left(Y\left(x_{0}, r\right), P\left(x_{0}, 4 r\right)\right)\right\}-8 \varepsilon r, 2 r\right\} \\
& \geq \min \{\min \{r / 2-2 \varepsilon r, r / 2\}-8 \varepsilon r, 2 r\}=\frac{r}{2}-10 \varepsilon r \geq \frac{29}{60} r .
\end{aligned}
$$

Finally, by comparing (2.10) and (2.8) we obtain (2.7).

$\diamond$ Step 5. We now establish (2.7) in case (II). If $z \in\left[x, Y\left(x_{0}, 2^{-k_{0}} r\right]\right.$, then we can repeat the argument we used in Step 4 by replacing $r$ with $2^{-k_{0}} r$, which satisfies

$$
d\left(x, \Omega^{c}\right) \leq 2^{-k_{0}} r \leq 2 d\left(x, \Omega^{c}\right) .
$$

Hence, we are left to consider the case when $z \in\left[Y\left(x_{0}, 2^{-k} r\right), Y\left(x_{0}, 2^{-(k+1)} r\right)\right]$ for some natural number $k \leq k_{0}-1$. We set $\rho:=2^{-k} r$ and we work in the ball $B\left(x_{0}, 2 \rho\right)$. We denote by $\alpha$ the angle between $\nu_{2 \rho}$ and $\nu_{\rho}$, and by $\beta$ the angle between $\nu_{2 \rho}$ and $\nu_{\rho / 2}$. Due to Lemma 5 applied with $M=2$ and $M=4$, we know that, if $\varepsilon \leq 1 / 13$, then

$$
\rho \cos \alpha \geq 4 \varepsilon \rho, \quad \frac{1}{2} \rho \cos \beta \geq 4 \varepsilon \rho,
$$

so that both $Y\left(x_{0}, \rho\right)$ and $Y\left(x_{0}, \rho / 2\right)$ belong to $B^{+}\left(x_{0}, 2 \rho\right)$. Hence, given

$$
z \in\left[Y\left(x_{0}, \rho\right), Y\left(x_{0}, \rho / 2\right)\right] \subseteq B^{+}\left(x_{0}, 2 \rho\right) \subseteq \Omega,
$$

we have $d\left(z, \Omega^{c}\right) \geq d\left(z, \partial B^{+}\left(x_{0}, 2 \rho\right)\right)$. The distance from $z$ to the spherical part of $\partial B^{+}\left(x_{0}, 2 \rho\right)$ is bounded from below by $\rho$, while the distance from $z$ to $P^{+}\left(x_{0}, 2 \rho\right)$ is 
bounded from below by $\frac{1}{2} \rho-4 \varepsilon \rho \geq \frac{1}{4} \rho$ provided that $\varepsilon \leq 1 / 16$. Hence, $d\left(z, \Omega^{c}\right) \geq \rho / 4$. To provide an upper bound on $d(z, x)$ we observe that, since $d\left(x, x_{0}\right)=d\left(x, \Omega^{c}\right) \leq$ $2^{-k} r$, then both $z$ and $x$ belong to the closure of $B\left(x_{0}, \rho\right)$. Hence, $d(x, z) \leq 2 \rho$ and (2.7) holds.

$\diamond$ Step 6. We are finally ready to show that $\Omega$ is Jones-flat. Given $x, y \in \Omega$ satisfying $d(x, y) \leq r_{0} / 7$, there are two possible cases:

(1) If either $d\left(x, \Omega^{c}\right) \geq 2 d(x, y)$ or $d\left(y, \Omega^{c}\right) \geq 2 d(x, y)$, then we set $\gamma:=[x, y]$. To see that $\gamma$ satisfies $(2.2)$, let us assume that $d\left(x, \Omega^{c}\right) \geq 2 d(x, y)$ (the other case is completely analogous), then $y \in B(x, d(x, y)) \subseteq \Omega$ and $[x, y] \subseteq \Omega$. Also, since

$$
\sup _{z \in[x, y]} \frac{d(z, x) d(z, y)}{d(x, y)}=\frac{1}{4} d(x, y),
$$

then for any $z \in \gamma$,

$$
d\left(z, \Omega^{c}\right) \geq d\left(x, \Omega^{c}\right)-d(z, x) \geq d(x, y) \geq 4 d(z, x) d(z, y) / d(x, y) .
$$

Hence, $\gamma$ satisfies $(2.2)$ provided that $\delta=4$.

(2) We are left to consider the case when both $d\left(x, \Omega^{c}\right)<2 d(x, y)$ and $d\left(y, \Omega^{c}\right)<$ $2 d(x, y)$. Denote by $x_{0} \in \partial \Omega$ a point such that $d\left(x, \Omega^{c}\right)=d\left(x, x_{0}\right)$ and $y_{0} \in \partial \Omega$ a point such that $d\left(y, y_{0}\right)=d\left(y, \Omega^{c}\right)$ and set $r:=d(x, y) \leq r_{0} / 7$. We define

$$
\gamma:=\gamma_{x, r} \cup \gamma_{y, r} \cup\left[Y\left(x_{0}, r\right), Y\left(y_{0}, r\right)\right] .
$$

Step 7 is devoted to showing that $\gamma$ satisfies (2.1) and (2.2).

$\diamond$ Step 7. First, we establish (2.1): we observe that

$$
\begin{aligned}
& d\left(Y\left(x_{0}, r\right), Y\left(y_{0}, r\right)\right) \\
& \leq d\left(Y\left(x_{0}, r\right), x_{0}\right)+d\left(x_{0}, x\right)+d(x, y)+d\left(y, y_{0}\right)+d\left(y_{0}, Y\left(y_{0}, r\right)\right) \leq 7 r
\end{aligned}
$$

and hence by using (2.5)

$$
\mathcal{H}^{1}(\gamma) \leq \mathcal{H}^{1}\left(\gamma_{x, r}\right)+d\left(Y\left(x_{0}, r\right), Y\left(y_{0}, r\right)\right)+\mathcal{H}^{1}\left(\gamma_{y, r}\right) \leq 15 r
$$

which proves $(2.1)$.

Next, we establish (2.2): we denote by $d_{\gamma}$ the geodesic distance on the curve $\gamma$ and we observe that

$$
\frac{d(z, y)}{15 d(x, y)} \leq \frac{d_{\gamma}(z, y)}{d_{\gamma}(x, y)} \leq 1
$$

Hence, if $z \in \gamma_{x, r}$, then by using (2.7) we obtain

$$
d\left(z, \Omega^{c}\right) \geq \frac{29}{240} d(z, x) \geq \frac{29}{240 \cdot 15}\left(\frac{d(z, x) d(z, y)}{d(x, y)}\right)
$$

and we next observe $29 / 240 \cdot 15 \geq 5 / 60 \cdot 15=1 / 180$. Since the same argument works in the case when $z \in \gamma_{y, r}$, then we are left to establish (2.2) in the case when $z$ lies on the segment $\left[Y\left(x_{0}, r\right), Y\left(y_{0}, r\right)\right]$.

We first observe that

$$
d\left(x_{0}, Y\left(y_{0}, r\right)\right) \leq d\left(x_{0}, x\right)+d(x, y)+d\left(y, y_{0}\right)+d\left(y_{0}, Y\left(y_{0}, r\right)\right) \leq 6 r
$$


and hence $\left[Y\left(x_{0}, r\right), Y\left(y_{0}, r\right)\right] \subseteq B\left(x_{0}, 7 r\right)$. Next, we note that $7 r \leq r_{0}$ and we use (2.10) to get

$$
\frac{29}{60} r \leq d\left(Y\left(x_{0}, r\right), \Omega^{c}\right) \leq d\left(Y\left(x_{0}, r\right), P^{-}\left(x_{0}, 7 r\right)\right)
$$

hence since $\varepsilon$ is so small that $28 \varepsilon r \leq 29 r / 60$, then we have $d\left(Y\left(x_{0}, r\right), P^{-}\left(x_{0}, 7 r\right)\right) \geq$ $28 \varepsilon r$, which means that $Y\left(x_{0}, r\right) \in B^{+}\left(x_{0}, 7 r\right)$. By repeating the same argument we get $Y\left(x_{0}, r\right) \in B^{+}\left(x_{0}, 7 r\right)$ and hence $\left[Y\left(x_{0}, r\right), Y\left(y_{0}, r\right)\right] \subseteq B^{+}\left(x_{0}, 7 r\right)$.

We fix $z \in\left[Y\left(x_{0}, r\right), Y\left(y_{0}, r\right)\right]$ and we observe that

$$
\begin{aligned}
d(z, x) & \leq d\left(z, Y\left(x_{0}, r\right)\right)+d\left(Y\left(x_{0}, r\right), x_{0}\right)+d\left(x_{0}, x\right) \\
& \leq d\left(Y\left(y_{0}, r\right), Y\left(x_{0}, r\right)\right)+d\left(Y\left(x_{0}, r\right), x_{0}\right)+d\left(x_{0}, x\right) \leq 7 r+r+2 r \\
& =10 r .
\end{aligned}
$$

Also,

$$
d\left(z, \Omega^{c}\right) \geq d\left(z, \partial B^{+}\left(x_{0}, 7 r\right)\right) \geq \min \left\{d\left(z, \partial B\left(x_{0}, 7 r\right)\right) ; d\left(z, P^{+}\left(x_{0}, 7 r\right)\right)\right\}
$$

and by using (2.14) we get

$$
d\left(z, \partial B\left(x_{0}, 7 r\right)\right) \geq r
$$

Also, we have

$$
d\left(z, P^{+}\left(x_{0}, 7 r\right)\right) \geq \min \left\{d\left(Y\left(x_{0}, r\right), P^{+}\left(x_{0}, 7 r\right)\right), d\left(Y\left(y_{0}, r\right), P^{+}\left(x_{0}, 7 r\right)\right)\right\}
$$

and by recalling $(2.15)$ we get that

$$
d\left(Y\left(x_{0}, r\right), P^{+}\left(x_{0}, 7 r\right)\right)=d\left(Y\left(x_{0}, r\right), P^{-}\left(x_{0}, 7 r\right)\right)-28 \varepsilon r \geq \frac{29}{60} r-28 \varepsilon r \geq \frac{r}{3} .
$$

Since $Y\left(y_{0}, r\right)$ satisfies the same estimate, then by recalling (2.13), (2.16) and (2.17) we get

$$
d\left(z, \Omega^{c}\right) \geq \frac{r}{3} \geq \frac{1}{3 \cdot 10} d(z, x) \geq \frac{1}{3 \cdot 10 \cdot 15} \frac{d(z, x) d(z, y)}{d(x, y)},
$$

which concludes the proof because $3 \cdot 10 \cdot 15=450$.

Remark 7. Note that there are open sets that are Jones-flat but not Reifenbergflat, for instance triangles. To get a flavour of the difference between Reifenbergflatness and Jones-flatness, we focus on dimension $N=2$. Jones [19, Theorem 4] proved that, if $\Omega \subseteq \mathbf{R}^{2}$ is a bounded and connected open set, then the following conditions are equivalent: i) $\Omega$ admits an extension operator; ii) $\Omega$ is $(\delta,+\infty)$-Jones flat for some $\delta>0$; iii) the boundary $\partial \Omega$ consists of a finite number of points and quasi-circles. We recall that a quasi-circle is the image of the unit circle under a quasi-conformal mapping, see the introduction of [19] for the precise definition. Conversely, Reifenberg Topological Disk Theorem [29] implies that, if $\Omega \subseteq \mathbf{R}^{2}$ is an $\left(\varepsilon, r_{0}\right)$-Reifenberg flat domain for a sufficiently small $\varepsilon>0$, then $\partial \Omega$ is locally the bi-Hölder image of a bounded interval. 


\section{Extension properties of Reifenberg-flat domains and applications}

In this section we combine the analysis in [19] with Theorem 3 to prove that open sets that are sufficiently flat in the sense of Reifenberg satisfy the extension property. We also discuss some direct consequences. Note that in this section we always assume that $\Omega$ is connected, as Jones did in [19]. In Section 4 we prove that the connectedness assumption can be actually removed in the case of Reifenbergflat-domain. Note also that, before providing the precise extension result, we have to introduce a preliminary lemma comparing different notions of "radius" of a given set $\Omega$.

3.1. "Inner radius", "outer radius" and "diameter" of a given set. We term outer radius of a nonempty set $\Omega \subseteq \mathbf{R}^{N}$ the quantity

$$
\operatorname{Rad}(\Omega):=\inf _{x \in \Omega} \sup _{y \in \Omega} d(x, y),
$$

and we term inner radius the quantity

$$
\operatorname{rad}(\Omega):=\sup _{x \in \Omega} \sup \{r>0: B(x, r) \subseteq \Omega\} .
$$

The inner radius is the radius of the biggest ball that could fit inside $\Omega$, whereas the outer radius, as seen below, is the radius of the smallest ball, centered in $\bar{\Omega}$, that contains $\Omega$.

Also, we recall that $\operatorname{Diam}(\Omega)$ denotes the diameter of $\Omega$, namely

$$
\operatorname{Diam}(\Omega):=\sup _{x, y \in \Omega} d(x, y) .
$$

For the convenience of the reader, we collect some consequences of the definition in the following lemma.

Lemma 8. Let $\Omega$ be a nonempty subset of $\mathbf{R}^{N}$, then the following properties hold:

(i) We have the formula

$$
\operatorname{Rad}(\Omega)=\inf _{x \in \Omega} \inf \{r>0: \Omega \subseteq B(x, r)\} .
$$

Also, if $\operatorname{Rad}(\Omega)<+\infty$, then there is a point $x \in \bar{\Omega}$ such that $\Omega \subseteq$ $B(x, \operatorname{Rad}(\Omega))$.

(ii) $\operatorname{rad}(\Omega) \leq \operatorname{Rad}(\Omega) \leq \operatorname{Diam}(\Omega)$.

(iii) If $\Omega$ is open and $\left(\varepsilon, r_{0}\right)$-Reifenberg-flat for some $r_{0}>0$ and some $\varepsilon$ satisfying $0<\varepsilon<1 / 2$, then $r_{0} / 4 \leq \operatorname{rad}(\Omega) \leq \operatorname{Rad}(\Omega) \leq \operatorname{Diam}(\Omega)$.

Proof. To establish property (i), we first observe that, if $\Omega$ is not bounded, then $\operatorname{Rad}(\Omega)=+\infty$ and formula (3.3) is trivially satisfied. Also, the assumption $\operatorname{Rad}(\Omega)<+\infty$ implies that the closure $\bar{\Omega}$ is compact. Hence, if $\operatorname{Rad}(\Omega)<+\infty$, then

$$
\operatorname{Rad}(\Omega)=\min _{x \in \bar{\Omega}} \sup _{y \in \Omega} d(x, y)
$$

and if we term $x_{0} \in \bar{\Omega}$ any point that realizes the minimum in (3.4) we have $\Omega \subseteq$ $\bar{B}\left(x_{0}, \operatorname{Rad}(\Omega)\right)$. This establishes the inequality

$$
\operatorname{Rad}(\Omega) \geq \inf _{x \in \Omega} \inf \{r>0: \Omega \subseteq B(x, r)\} .
$$


To establish the reverse inequality we observe that if $x \in \Omega$ is any arbitrary point and $r>0$ is such that $\Omega \subseteq B(x, r)$, then $\sup _{y \in \Omega} d(x, y) \leq r$. By taking the infimum in $x$ and $r$ we conclude. This ends the proof of property (i).

To establish (ii), we focus on the case when $\operatorname{Rad}(\Omega)<+\infty$, because otherwise $\Omega$ is unbounded and (ii) trivially holds. Hence, by relying on (i) we infer that $\Omega \subseteq B:=$ $B\left(x_{0}, \operatorname{Rad}(\Omega)\right)$ for some point $x_{0} \in \Omega$. Given $x \in \Omega$ and $r>0$ satisfying $B(x, r) \subseteq \Omega$, we have $B(x, r) \subseteq B\left(x_{0}, \operatorname{Rad}(\Omega)\right)$. Hence, $d\left(x, x_{0}\right)+r \leq \operatorname{Rad}(\Omega)$ and hence $r \leq$ $\operatorname{Rad}(\Omega)$. By taking the supremum in $r$ and $x$ we get finally $\operatorname{rad}(\Omega) \leq \operatorname{Rad}(\Omega)$. The inequality $\operatorname{Rad}(\Omega) \leq \operatorname{Diam}(\Omega)$ directly follows from the two definitions.

Given (ii), establishing property (iii) amounts to show that

$$
\operatorname{rad}(\Omega) \geq r_{0} / 4
$$

We can assume with no loss of generality that $\partial \Omega \neq \emptyset$, otherwise $\Omega=\mathbf{R}^{N}$ and (3.5) trivially holds in this case (we recall that the case $\Omega=\emptyset$ is ruled out by the definition of Reifenberg-flatness).

Hence, we fix $y \in \partial \Omega$, denote by $P\left(y, r_{0}\right)$ the hyperplane in the definition and let $\vec{\nu}$ be its normal vector. We choose the orientation of $\vec{\nu}$ in such a way that

$$
\left\{z+t \nu: z \in P\left(y, r_{0}\right), t \geq 2 \varepsilon r\right\} \cap B\left(y, r_{0}\right) \subseteq \Omega .
$$

Since $d_{H}\left(P\left(y, r_{0}\right) \cap B\left(y, r_{0}\right), \partial \Omega \cap B\left(y, r_{0}\right)\right) \leq \varepsilon r$, then from (3.6) we infer that actually

$$
\left\{z+t \nu: z \in P\left(y, r_{0}\right), t \geq \varepsilon r\right\} \cap B\left(y, r_{0}\right) \subseteq \Omega .
$$

By recalling $\varepsilon<1 / 2$, we infer that there is $x \in \Omega$ such that $B\left(x, r_{0} / 4\right) \subseteq \Omega$ and this establishes (3.5).

3.2. Extension properties and applications. The following extension property for Reifenberg flat domains is established by combining Theorem 3 above with Jones'analysis (Theorem 1 in [19]).

Corollary 9. Let $\Omega \subseteq \mathbf{R}^{N}$ be a connected, $\left(\varepsilon, r_{0}\right)$-Reifenberg-flat-domain. If $\varepsilon \leq 1 / 600$, then, for every $p \in[1,+\infty]$, there is an extension operator $E: W^{1, p}(\Omega) \rightarrow$ $W^{1, p}\left(\mathbf{R}^{N}\right)$ satisfying

$$
\|E(u)\|_{W^{1, p}\left(\mathbf{R}^{N}\right)} \leq C\|u\|_{W^{1, p}(\Omega)},
$$

where the constant $C$ only depends on $N, p$, and $r_{0}$.

Proof. The corollary is a direct application of [19, Theorem 1].

The only nontrivial point we have to address is that, in general, the norm of the extension operator $E$ depends on $\operatorname{Rad}(\Omega)$, see for examples the statements of Jones Theorem provided in the paper by Chua [7] and in the very recent preprint by Brewster, D. Mitrea, I. Mitrea and M. Mitrea [1]. Note that in Jones' original statement the dependence on the radius was not mentioned because the radius was fixed (see the remark at the top of page 76 in [19]).

However, by applying for example the remarks in [1, pages 9 and 10], we get that the norm of $E$ is bounded by $C\left(N, p, r_{0}, M\right)$ if $1 / \operatorname{Rad}(\Omega) \leq M$. By recalling that $r_{0} / 4 \leq \operatorname{Rad}(\Omega)$, we finally infer that the bound on the norm of the extension operator only depends on $N, p$ and $r_{0}$ and this concludes the proof.

Remark 10. To simplify the exposition, we chose to only state the extension property for classical Sobolev Spaces. However, the extension property also applies to other classes of spaces. For instance, Chua [7, 8, 9], extended Jones Theorem to 
weighted Sobolev spaces. These spaces are defined by replacing the Lebesgue measure by a weighted measure $\omega d x$, where $\omega$ is a function satisfying suitable growth conditions and Poincaré inequalities. Also, Christ [6] established the extension property for Sobolev spaces of fractional order.

The results of both Christ [6] and Chua [7, 8, 9] apply to Jones-flat domains, hence by relying on Theorem 3 we infer that they apply to $\left(1 / 600, r_{0}\right)$-Reifenbergflat-domains as well.

As a consequence of Corollary 9 we get that the classical Rellich-Kondrachov Theorem holds in Reifenberg-flat-domains. For other results concerning extensions of the Rellich-Kondrachov Theorem to rather irregular domains, we refer to the paper by Hajłasz and Koskela [15] and to the references therein.

Proposition 11. Let $\Omega \subseteq \mathbf{R}^{N}$ be a bounded, connected $\left(\varepsilon, r_{0}\right)$-Reifenberg-flatdomain and assume $0<\varepsilon \leq 1 / 600$.

If $1 \leq p<N$, set $p^{*}:=\frac{N p}{N-p}$. Then the Sobolev space $W^{1, p}(\Omega)$ is continuously embedded in the space $L^{p^{*}}(\Omega)$ and is compactly embedded in $L^{q}(\Omega)$ for every $1 \leq$ $q<p^{*}$.

If $p \geq N$, then the Sobolev space $W^{1, N}(\Omega)$ is continuously embedded in the space $L^{\infty}(\Omega)$ and is compactly embedded in $L^{q}(\Omega)$ for every $q \in[1,+\infty[$.

Also, the norm of the above embedding operators only depends on $N, r_{0}, q, p$ and $\operatorname{Rad}(\Omega)$.

Proof. We first use the extension operator provided by Corollary 9 and then we apply the classical Embedding Theorem in a ball of $\operatorname{radius} \operatorname{Rad}(\Omega)$ containing $\Omega$ (see property (i) in the statement of Lemma 8).

As an example of application of Proposition 11, we establish a uniform bound on the $L^{\infty}$ norm of Neumann eigenfunctions defined in Reifenberg-flat domains. We use this bound in the companion paper [27]. Here is the precise statement. We recall that we term "Neumann eigenfunction" an eigenfunction for the Laplace operator subject to homogeneous Neumann conditions on the boundary of the domain.

Proposition 12. Let $\Omega \subseteq \mathbf{R}^{N}$ be a bounded, connected, $\left(\varepsilon, r_{0}\right)$-Reifenberg-flatdomain and let $u$ be a Neumann eigenfunction associated to the eigenvalue $\mu$. If $\varepsilon \leq 1 / 600$, then $u$ is bounded and

$$
\|u\|_{L^{\infty}(\Omega)} \leq C(1+\sqrt{\mu})^{\gamma(N)}\|u\|_{L^{2}(\Omega)}
$$

where $\gamma(N)=\max \left\{\frac{N}{2}, \frac{2}{N-1}\right\}$ and $C=C\left(N, r_{0}, \operatorname{Rad}(\Omega)\right)$.

Proof. By using classical techniques coming from the regularity theory for elliptic operators, Ross [30, Proposition 3.1] established (3.7) in the case of Lipschitz domains. However, in [30] the only reason why one needs the regularity assumption on the domain $\Omega$ is to use the Sobolev inequality

$$
\|u\|_{L^{2^{*}(\Omega)}} \leq C\left(\|u\|_{L^{2}(\Omega)}+\|\nabla u\|_{L^{2}(\Omega)}\right), \quad C=C\left(N, r_{0}, \operatorname{Rad}(\Omega)\right)
$$

as the starting point for a bootstrap argument. Since Proposition 11 states that (3.8) holds if $\Omega$ is a bounded Reifenberg-flat domain, then the proof in [30] can be extended to the case when $\Omega$ is Reifenberg-flat. 
Remark 13. An inequality similar to (3.7) holds for Dirichlet eigenfunctions. We emphasize that the boundedness of Dirichlet eigenfunctions, unlike the boundedness of Neumann eigenfunctions, does not require any regularity assumption on the set $\Omega$, see for instance [14, Lemma 3.1] for a precise statement.

\section{Connected components of Reifenberg-flat domains}

In the previous section we have always assumed that the open set $\Omega$ is connected. We now show that the results we have established can be extended to general (i.e., not necessarily connected) Reifenberg-flat open sets. Although extension of the result of Jones [19] to non-connected sets were already widely known in the literature, we decided to provide here a self-contained proof. In this way, we obtain results on the structure of Reifenberg-flat-domains that may be of independent interest.

We first show that any bounded, open set that is sufficiently flat in the sense of Reifenberg has finitely many connected components and we establish a quantitative bound on the Hausdorff distance between two connected components.

Proposition 14. Let $\Omega \subseteq \mathbf{R}^{N}$ be a bounded, $\left(\varepsilon, r_{0}\right)$-Reifenberg-flat-domain and assume $\varepsilon \leq 20^{-N}$. Then $\Omega$ has a finite number of nonempty, open and disjoint connected components $U_{1}, \ldots, U_{n}$, where

$$
n \leq \frac{20^{N}}{\omega_{N}} \frac{|\Omega|}{r_{0}^{N}}
$$

Moreover, if $i \neq j$, then for every $z \in \partial U_{i}$ we have

$$
d\left(z, U_{j}\right)>r_{0} / 70 .
$$

Proof. We proceed according to the following steps.

$\diamond$ Step 1 . We recall that any nonempty open set $\Omega \subseteq \mathbf{R}^{N}$ can be decomposed as

$$
\Omega:=\bigcup_{i \in I} U_{i}
$$

where the connected components $U_{i}$ satisfy

- for every $i \in I, U_{i}$ is a nonempty, open, arcwise connected set which is also closed in $\Omega$. Hence, in particular, $\partial U_{i} \subseteq \partial \Omega$.

- $U_{i} \cap U_{j}=\emptyset$ if $i \neq j$.

Indeed, for any $x \in \Omega$ we can define

$$
\begin{array}{r}
U_{x}:=\{y \in \Omega: \text { there is a continuous curve } \gamma:[0,1] \rightarrow \Omega \\
\text { such that } \gamma(0)=x \text { and } \gamma(1)=y\}
\end{array}
$$

and observe that any $U_{x}$ is a nonempty, open, arcwise connected set which is also closed in $\Omega$. Also, given two points $x, y \in \mathbf{R}^{N}$, we have either $U_{x}=U_{y}$ or $U_{x} \cap U_{y}=\emptyset$.

$\diamond$ Step 2. Let $\Omega$ as in the statement of the proposition, and let the family $\left\{U_{i}\right\}_{i \in I}$ be as in (4.3). We fix $i \in I$ and we prove that $\left|U_{i}\right| \geq C\left(r_{0}, N\right)$. This straightforwardly implies that $\sharp I \leq C\left(|\Omega|, r_{0}, N\right)$.

Since $U_{i}$ is bounded, then $\partial U_{i} \neq \emptyset$ : hence, we can fix a point $\tilde{x} \in \partial U_{i}$, and a sequence $\left\{x_{n}\right\}_{n \in \mathbf{N}}$ such that $x_{n} \in U_{i}$ and $x_{n} \rightarrow \tilde{x}$ as $n \rightarrow+\infty$. We recall that $\partial U_{i} \subseteq \partial \Omega$ and we infer that, for any $n \in \mathbf{N}$, the following chain of inequalities holds:

$$
d\left(x_{n}, \partial U_{i}\right)=d\left(x_{n}, U_{i}^{c}\right) \leq d\left(x_{n}, \Omega^{c}\right)=d\left(x_{n}, \partial \Omega\right) \leq d\left(x_{n}, \partial U_{i}\right),
$$


which implies $d\left(x_{n}, \Omega^{c}\right)=d\left(x_{n}, \partial U_{i}\right)$. We fix $n$ sufficiently large such that $d\left(x_{n}, \tilde{x}\right) \leq$ $r_{0} / 7$, so that

$$
d\left(x_{n}, \Omega^{c}\right)=d\left(x_{n}, \partial U_{i}\right) \leq r_{0} / 7 .
$$

We term $\Gamma:=\gamma_{x_{n}, r_{0} / 7}$ the polygonal curve constructed as in Step 2 of the proof of Theorem 3 and we observe that, if $\varepsilon \leq 1 / 32$, then (2.7) holds and $\Gamma \subseteq \Omega$ and hence, by definition of $U_{i}, \Gamma \subseteq U_{i}$. We use the same notation as in Step 1 of the proof of Theorem 3 and we recall that $\Gamma$ connects $x_{n}$ to some point $Y\left(x_{0}, r_{0} / 7\right)$, defined with some $x_{0} \in \partial \Omega$. Hence, in particular, $Y\left(x_{0}, r_{0} / 7\right) \in U_{i}$ and this implies that $B^{+}\left(x_{0}, r_{0} / 7\right) \subseteq U_{i}$ because $B^{+}\left(x_{0}, r_{0} / 7\right)$ is connected. This finally yields

$$
\left|U_{i}\right| \geq\left|B^{+}\left(x_{0}, r_{0} / 7\right)\right| \geq \omega_{N}\left(\frac{r_{0}}{14}(1-2 \varepsilon)\right)^{N} \geq \omega_{N}\left(\frac{9 r_{0}}{140}\right)^{N} \geq \omega_{N}\left(\frac{r_{0}}{20}\right)^{N}
$$

because $\varepsilon \leq 1 / 20$. We deduce that

$$
\sharp I \leq \frac{20^{N}}{\omega_{N}} \frac{|\Omega|}{r_{0}^{N}} .
$$

$\diamond$ Step 3. We establish the separation property (4.2).

We set $r_{1}:=r_{0} / 70$ and we argue by contradiction, assuming that there are $z \in \partial U_{i}, y \in \partial U_{j}$ such that

$$
d\left(z, U_{j}\right)=d\left(z, \partial U_{j}\right)=d(z, y) \leq r_{1} .
$$

Let $\left\{z_{n}\right\}_{n \in \mathbf{N}}$ and $\left\{y_{n}\right\}_{n \in \mathbf{N}}$ be sequences in $U_{i}$ and $U_{j}$ converging to $z$ and $y$, respectively. We fix $n$ sufficiently large such that

$$
d\left(z_{n}, \partial U_{i}\right) \leq d\left(z_{n}, z\right) \leq r_{1} \leq r_{0} / 14
$$

and we term $\bar{z}$ be a point in $\partial U_{i}$ satisfying $d\left(z_{n}, \bar{z}\right)=d\left(z_{n}, \partial U_{i}\right)$ (if there is more than one such $\bar{z}$, we arbitrarily fix one). By arguing as in Step 2, we infer that $B^{+}\left(\bar{z}, r_{0} / 14\right) \subseteq U_{i}$. Next, we do the same for $U_{j}$, namely we fix $m$ sufficiently large that

$$
d\left(y_{m}, \partial U_{j}\right) \leq d\left(y_{m}, y\right) \leq r_{1} \leq r_{0} / 7
$$

we let $\bar{y}$ be a point in $\partial U_{j}$ satisfying $d\left(y_{m}, \bar{y}\right)=d\left(y_{m}, \partial U_{j}\right)$ and, by arguing as in STEP 2 , we get that $B^{+}\left(\bar{y}, r_{0} / 7\right) \subseteq U_{j}$. Also, we note that

$$
d(\bar{z}, \bar{y}) \leq d\left(\bar{z}, z_{n}\right)+d\left(z_{n}, z\right)+d(z, y)+d\left(y, y_{m}\right)+d\left(y_{m}, \bar{y}\right) \leq 5 r_{1} .
$$

Since $r_{1}=r_{0} / 70$, then $B^{+}\left(\bar{z}, r_{0} / 14\right) \subseteq B\left(\bar{z}, r_{0} / 14\right) \subseteq B\left(\bar{y}, r_{0} / 7\right)$. We observe that

$$
B^{+}\left(\bar{z}, r_{0} / 14\right) \cap B^{-}\left(\bar{y}, r_{0} / 7\right)=\emptyset
$$

since by construction $B^{+}\left(\bar{z}, r_{0} / 14\right) \subseteq \Omega$ and $B^{-}\left(\bar{y}, r_{0} / 7\right) \subseteq \Omega^{c}$. Also, by recalling that

$$
B^{+}\left(\bar{z}, r_{0} / 14\right) \subseteq U_{i}, \quad B^{+}\left(\bar{y}, r_{0} / 7\right) \subseteq U_{j} \quad \text { and } \quad U_{i} \cap U_{j}=\emptyset,
$$

we have that

$$
B^{+}\left(\bar{z}, r_{0} / 14\right) \cap B^{+}\left(\bar{y}, r_{0} / 7\right)=\emptyset .
$$

By combining (4.4) and (4.5) we get

$$
B^{+}\left(\bar{z}, r_{0} / 14\right) \subseteq B\left(\bar{y}, r_{0} / 7\right) \backslash\left(B^{+}\left(\bar{y}, r_{0} / 7\right) \cup B^{-}\left(\bar{y}, r_{0} / 7\right)\right)
$$

We now use the inequality

$$
\omega_{N} \geq \omega_{N-1} \frac{1}{2^{N-1}}
$$


which can be established by combining the formula

$$
\omega_{N}=\omega_{N-1} \int_{-1}^{1}\left(\sqrt{1-x^{2}}\right)^{N-1} d x
$$

with Jensen's inequality. By relying on (4.7) and by recalling that $\varepsilon \leq 20^{-N} \leq 1 / 20$ we obtain

$$
\left|B^{+}\left(\bar{z}, r_{0} / 14\right)\right| \geq \omega_{N}\left(\frac{r_{0}}{28}(1-2 \varepsilon)\right)^{N} \geq 2 \omega_{N-1}\left(\frac{9 r_{0}}{560}\right)^{N}
$$

and

$$
\left|B\left(\bar{y}, r_{0} / 7\right) \backslash\left(B^{+}\left(\bar{y}, r_{0} / 7\right) \cup B^{-}\left(\bar{y}, r_{0} / 7\right)\right)\right| \leq 4 \varepsilon \omega_{N-1}\left(\frac{r_{0}}{7}\right)^{N} \leq 2 \omega_{N-1}\left(\frac{2 r_{0}}{140}\right)^{N},
$$

which contradicts $(4.6)$ since $2 / 140<9 / 560$.

By relying on Proposition 14 we can now remove the connectedness assumption in the statement of Proposition 9.

Corollary 15. Let $N \geq 2$ and $\Omega \subseteq \mathbf{R}^{N}$ be a bounded, $\left(\varepsilon, r_{0}\right)$-Reifenberg-flatdomain with $\varepsilon \leq \min \left(20^{-N}, 1 / 600\right)$. Then for every $p \in[1,+\infty]$ there is an extension operator

$$
E: W^{1, p}(\Omega) \rightarrow W^{1, p}\left(\mathbf{R}^{N}\right)
$$

whose norm is bounded by a constant which only depends on $N, p$, and $r_{0}$.

Proof. We employ the same notation as in the statement of Proposition 14 and we fix a connected component $U_{i}$. By recalling that $\partial U_{i} \subseteq \partial \Omega$ and the separation property (4.2), we infer that $U_{i}$ is itself $\left(\varepsilon, r_{0} / 140\right)$-Reifenberg flat. Since by definition $U_{i}$ is connected, we can apply Proposition 9 which says that, for every $p \in[1,+\infty]$, there is an extension operator

$$
E_{i}: W^{1, p}\left(U_{i}\right) \rightarrow W^{1, p}\left(\mathbf{R}^{N}\right)
$$

whose norm is bounded by a constant which only depends on $N, p$ and $r_{0}$.

In order to "glue together" the extension operators $E_{1}, \ldots, E_{n}$ we proceed as follows. Given $i=1, \ldots, n$, we set $\delta:=r_{0} / 280$ and we introduce the notation

$$
U_{i}^{\delta}:=\left\{x \in \mathbf{R}^{N}: d\left(x, U_{i}\right)<\delta\right\} .
$$

Note that the separation property (4.2) implies that $U_{i}^{2 \delta} \cap U_{j}^{2 \delta}=\emptyset$ if $i \neq j$.

We now construct suitable cut-off functions $\varphi_{i}, i=1, \ldots, n$. Let $\ell:[0,+\infty[\rightarrow$ $[0,1]$ be the auxiliary function defined by setting

$$
\ell(t):= \begin{cases}1 & \text { if } t \leq \delta \\ 1+\frac{\delta-t}{\delta} & \text { if } \delta \leq t \leq 2 \delta \\ 0 & \text { if } t \geq 2 \delta .\end{cases}
$$

We set $\varphi_{i}(x):=\ell\left(d\left(x, U_{i}\right)\right)$ and we recall that the function $x \mapsto d\left(x, U_{i}\right)$ is 1-Lipschitz and that $\delta=r_{0} / 280$. Hence, the function $\varphi_{i}$ satisfies the following properties:

$$
\begin{aligned}
& 0 \leq \varphi_{i}(x) \leq 1, \quad\left|\nabla \varphi_{i}(x)\right| \leq C\left(r_{0}\right) \forall x \in \mathbf{R}^{N}, \\
& \varphi_{i} \equiv 1 \text { on } U_{i}, \quad \varphi_{i} \equiv 0 \text { on } \mathbf{R}^{N} \backslash U_{i}^{2 \delta}
\end{aligned}
$$


We then define $E: W^{1, p}(\Omega) \rightarrow W^{1, p}\left(\mathbf{R}^{N}\right)$ by setting

$$
E(u):=\sum_{i=1}^{n} E_{i}(u)(x) \varphi_{i}(x) .
$$

We recall that the sets $U_{1}, \ldots, U_{n}$ are all pairwise disjoint, we focus on the case $p<+\infty$ and we get

$$
\begin{aligned}
\|E(u)\|_{L^{p}\left(\mathbf{R}^{N}\right)} & =\left(\int_{\mathbf{R}^{N}}\left|\sum_{i=1}^{n} E_{i}(u)(x) \varphi_{i}(x) d x\right|^{p}\right)^{1 / p} \\
& \leq \sum_{i=1}^{n}\left(\int_{U_{i}^{2 \delta}}\left|E_{i}(u)(x) \varphi_{i}(x)\right|^{p} d x\right)^{1 / p} \leq \sum_{i=1}^{n}\left\|E_{i}(u)\right\|_{L^{p}\left(\mathbf{R}^{N}\right)} \\
& \leq \sum_{i=1}^{n} C\left(N, p, r_{0}\right)\|u\|_{W^{1, p}\left(U_{i}\right)} \leq C\left(N, p, r_{0}\right)\|u\|_{W^{1, p}(\Omega)} .
\end{aligned}
$$

Also, by using the bound on $\left|\nabla \varphi_{i}\right|$ provided by (4.9), we get

$$
\begin{aligned}
& \|\nabla E(u)\|_{L^{p}\left(\mathbf{R}^{N}\right)}=\left(\int_{\mathbf{R}^{N}}\left|\sum_{i=1}^{n}\left(\nabla E_{i}(u)(x) \varphi_{i}(x)+E_{i}(u)(x) \nabla \varphi_{i}(x)\right) d x\right|^{p}\right)^{1 / p} \\
& \leq \sum_{i=1}^{n}\left(\int_{U_{i}^{2 \delta}}\left|\nabla E_{i}(u)(x) \varphi_{i}(x)\right|^{p} d x\right)^{1 / p}+\sum_{i=1}^{n}\left(\int_{U_{i}^{2 \delta}}\left|E_{i}(u)(x) \nabla \varphi_{i}(x)\right|^{p} d x\right)^{1 / p} \\
& \leq \sum_{i=1}^{n}\left\|\nabla E_{i}(u)\right\|_{L^{p}\left(\mathbf{R}^{N}\right)}+C\left(r_{0}\right) \sum_{i=1}^{n}\left\|E_{i}(u)\right\|_{L^{p}\left(\mathbf{R}^{N}\right)} \leq C\left(N, p, r_{0}\right)\|u\|_{W^{1, p}(\Omega)} .
\end{aligned}
$$

The proof in the case $p=\infty$ is a direct consequence of the bounds on the norm of $E_{i}$ and on the uniform norms of $\varphi_{i}$ and $\nabla \varphi_{i}$. This concludes the proof of the corollary.

\section{On the Hausdorff distance between Reifenberg-flat domains}

We end this paper by comparing different ways of measuring the "distance" between Reifenberg-flat-domains.

5.1. Comparison between different Hausdorff distances. This subsections aims at comparing the Hausdorff distances $d_{H}(X, Y), d_{H}\left(X^{c}, Y^{c}\right)$ and $d_{H}(\partial X, \partial Y)$, where $X$ and $Y$ are subsets of $\mathbf{R}^{N}$.

First, we exhibit two examples showing that, in general, neither $d_{H}(X, Y)$ controls $d_{H}\left(X^{c}, Y^{c}\right)$ nor $d_{H}\left(X^{c}, Y^{c}\right)$ controls $d_{H}(X, Y)$. We term $B:=B(\overrightarrow{0}, 1)$ the unit ball and we consider the two perturbations $A$ and $C$ as represented in Figure 2.

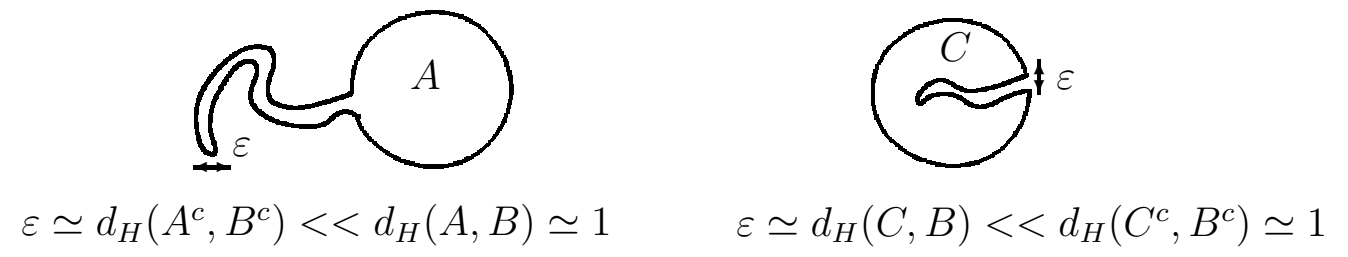

Figure 2. 
Next, we exhibit an example showing that, in general, $d_{H}(\partial X, \partial Y)$ controls neither $d_{H}(X, Y)$ nor $d_{H}\left(X^{c}, Y^{c}\right)$. Let $X:=B(\overrightarrow{0}, R)$ and $Y:=B(\overrightarrow{0}, R+\varepsilon) \backslash B(\overrightarrow{0}, R)$, then

$$
\varepsilon=d_{H}(\partial X, \partial Y)<<d_{H}(X, Y)=d_{H}\left(X^{c}, Y^{c}\right)=R .
$$

Also, note that the examples represented in Figure 2 show that, in general, neither $d_{H}(X, Y)$ nor $d_{H}\left(X^{c}, Y^{c}\right)$ controls $d_{H}(\partial X, \partial Y)$. Indeed, $d_{H}(\partial A, \partial B) \simeq 1$ and $d_{H}(\partial C, \partial B) \simeq 1$.

However, if $X$ and $Y$ are two sufficiently close Reifenberg-flat-domains, then we have the following result.

Lemma 16. Let $X$ and $Y$ be two $\left(\varepsilon, r_{0}\right)$-Reifenberg-flat-domains satisfying $d_{H}(\partial X, \partial Y) \leq 2 r_{0}$. Then

$$
d_{H}(\partial X, \partial Y) \leq \frac{4}{1-2 \varepsilon} \min \left\{d_{H}(X, Y), d_{H}\left(X^{c}, Y^{c}\right)\right\} .
$$

Proof. Just to fix the ideas, assume that $d_{H}(\partial X, \partial Y)=\sup _{x \in \partial X} d(x, \partial Y)$. Since by assumption $d_{H}(\partial X, \partial Y)<+\infty$, then for every $h>0$ there is $x_{h} \in \partial X$ such that

$$
d_{H}(\partial X, \partial Y)-h \leq d_{h}:=d\left(x_{h}, \partial Y\right) \leq d_{H}(\partial X, \partial Y) .
$$

Note that $\partial Y \cap B\left(x_{h}, d_{h} / 2\right)=\emptyset$ and hence either (i) $B\left(x_{h}, d_{h} / 2\right) \subseteq Y$ or (ii) $B\left(x_{h}\right.$, $\left.d_{h} / 2\right) \subseteq Y^{c}$.

First, consider case (i): let $P\left(x_{h}, d_{h} / 2\right)$ be the hyperplane prescribed by the definition of Reifenberg flatness, then by Lemma 6 we can choose the orientation of the normal vector $\nu$ in such a way that

$$
B^{-}\left(x_{h}, d_{h} / 2\right):=\left\{z+t \nu: z \in P\left(x_{h}, d_{h} / 2\right), t \geq \varepsilon d_{h}\right\} \cap B\left(x_{h}, d_{h} / 2\right) \subseteq X^{c}
$$

and

$$
B^{+}\left(x_{h}, d_{h} / 2\right):=\left\{z-t \nu: z \in P\left(x_{h}, d_{h} / 2\right), t \geq \varepsilon d_{h}\right\} \cap B\left(x_{h}, d_{h} / 2\right) \subseteq X .
$$

Fix the point

$$
\bar{z}:=x_{h}+\frac{(1+2 \varepsilon) d_{h}}{4} \nu
$$

then we have

$$
B\left(\bar{z}, \frac{(1-2 \varepsilon) d_{h}}{4}\right) \subseteq B^{-}\left(x_{h}, d_{h} / 2\right) \subseteq X^{c} \cap Y
$$

and hence

$$
d_{H}\left(X^{c}, Y^{c}\right) \geq \sup _{z \in X^{c}} d\left(z, Y^{c}\right) \geq d\left(\bar{z}, Y^{c}\right) \geq \frac{(1-2 \varepsilon) d_{h}}{4}
$$

and

$$
d_{H}(X, Y) \geq \sup _{z \in Y} d(z, X) \geq d(\bar{z}, X) \geq \frac{(1-2 \varepsilon) d_{h}}{4} .
$$

Since case (ii) can be tackled in an entirely similar way, by the arbitrariness of $h$ we deduce that

$$
d_{H}(\partial X, \partial Y) \leq \frac{4}{1-2 \varepsilon} d_{H}(X, Y)
$$

The proof of (5.1) is concluded by making the following observations:

- If $X$ is $\left(\varepsilon, r_{0}\right)$-Reifenberg flat, then $X^{c}$ is also $\left(\varepsilon, r_{0}\right)$-Reifenberg flat.

- $\partial X=\partial X^{c}$ and $\partial Y=\partial Y^{c}$. 
Hence, by replacing in (5.2) $X$ with $X^{c}$ and $Y$ with $Y^{c}$ we obtain (5.1).

5.2. Comparison between the Hausdorff distance and the measure of the symmetric difference. This subsection aims at comparing the Hausdorff distances $d_{H}(X, Y)$ and $d_{H}\left(X^{c}, Y^{c}\right)$ with the Lebesgue measure of the symmetric difference, $|X \triangle Y|$. As usual, $X$ and $Y$ are subsets of $\mathbf{R}^{N}$. The results we state are applied in [27] to the stability analysis of the spectrum of the Laplace operator with Neumann boundary conditions.

First, we observe that the examples illustrated in Figure 2 show that, in general, $|X \triangle Y|$ controls neither $d_{H}(X, Y)$ nor $d_{H}\left(X^{c}, Y^{c}\right)$. Indeed, $|A \triangle B| \simeq \varepsilon$ and $|C \triangle B| \simeq$ $\varepsilon$. However, if $X$ and $Y$ are two sufficiently close Reifenberg-flat-domains, then the following result hold.

Lemma 17. Let $X$ and $Y$ be two $\left(\varepsilon, r_{0}\right)$-Reifenberg-flat-domains in $\mathbf{R}^{N}$. Then the following implications hold:

(1) if $d_{H}(X, Y) \leq 4 r_{0}$, then

$$
d_{H}(X, Y) \leq \frac{8}{(1-2 \varepsilon)}\left(\frac{|X \triangle Y|}{\omega_{N}}\right)^{1 / N} .
$$

(2) If $d_{H}\left(X^{c}, Y^{c}\right) \leq 4 r_{0}$, then

$$
d_{H}\left(X^{c}, Y^{c}\right) \leq \frac{8}{(1-2 \varepsilon)}\left(\frac{|X \triangle Y|}{\omega_{N}}\right)^{1 / N} .
$$

In both the previous expressions, $\omega_{N}$ denotes the measure of the unit ball in $\mathbf{R}^{N}$.

Proof. The argument relies on ideas similar to those used in the proof of Lemma 16. We first establish (5.3). Just to fix the ideas, assume that $d_{H}(X, Y)=$ $\sup _{x \in X} d(x, Y)$ and note that by assumption $d_{H}(X, Y)<+\infty$. Hence, for every $h>0$ there is $x_{h} \in X$ such that

$$
d_{H}(X, Y)-h \leq d_{h}:=d\left(x_{h}, Y\right) \leq d_{H}(X, Y) .
$$

Note that, by the very definition of $d\left(x_{h}, Y\right)$, we have $B\left(x_{h}, d_{h}\right) \subseteq Y^{c}$. We now separately consider two cases: if $B\left(x_{h}, d_{h} / 2\right) \subseteq X$, then

$$
B\left(x_{h}, d_{h} / 2\right) \subseteq X \cap Y^{c} \subseteq|X \triangle Y|
$$

and hence

$$
\omega_{N}\left(\frac{d_{h}}{2}\right)^{N} \leq|X \triangle Y|,
$$

and by the arbitrariness of $h$ this implies (5.3).

Hence, we are left to consider the case when there is $x_{0} \in B\left(x_{h}, d_{h} / 2\right) \cap \partial X$. We make the following observations: first,

$$
B\left(x_{0}, d_{h} / 4\right) \subseteq B\left(x_{h}, d_{h}\right) \subseteq Y^{c} .
$$

Second, since $d_{h} / 4 \leq d_{H}(X, Y) / 4 \leq r_{0}$, then we can apply the definition of Reifenberg-flatness in the ball $B\left(x_{0}, d_{h} / 4\right)$. Let $P\left(x_{0}, d_{h} / 4\right)$ be the hyperplane provided by property (i) in the definition, and let $\nu_{0}$ denote the normal vector. By relying on Lemma 6 we infer that we can choose the orientation of $\nu_{0}$ in such a way that

$$
B\left(x_{0}+\frac{(1+2 \varepsilon) d_{h}}{8} \nu_{0}, \frac{(1-2 \varepsilon) d_{h}}{8}\right) \subseteq X \cap B\left(x_{0}, d_{h} / 4\right) .
$$


By combining (5.5) and (5.6) we infer that

$$
\omega_{N}\left(\frac{(1-2 \varepsilon) d_{h}}{8}\right)^{N} \leq\left|X \cap Y^{c}\right| \leq|X \triangle Y|
$$

and by the arbitrariness of $h$ this completes the proof of (5.3).

Estimate (5.4) follows from (5.3) by relying on the following two observations:

- $X \triangle Y=\left(X^{c} \cap Y\right) \cup\left(X \cap Y^{c}\right)=X^{c} \triangle Y^{c}$.

- If $X$ is $\left(\varepsilon, r_{0}\right)$-Reifenberg flat, then $X^{c}$ is also $\left(\varepsilon, r_{0}\right)$-Reifenberg flat.

Hence, by replacing in (5.3) $X$ with $X^{c}$ and $Y$ with $Y^{c}$ we get (5.4).

Acknowledgements. The authors wish to thank Tatiana Toro and Guy David for several conversations on Reifenberg flat sets. They also thank the anonymous referees for their remarks that helped improve the exposition. E. Milakis was supported by the Marie Curie International Reintegration Grant No 256481 within the 7th European Community Framework Programme. Part of this work was done when L. V. Spinolo was affiliated to the University of Zurich, Switzerland, which she thanks for the kind hospitality. A. Lemenant has been supported by the Agence National de la Recherche, through the project ANR-12-BS01-0014-01 GEOMETRYA.

\section{References}

[1] Brewster, K., D. Mitrea, I. Mitrea, and M. Mitrea: Extending Sobolev functions with partially vanishing traces from locally (epsilon,delta)-domains and applications to mixed boundary problems. - Preprint, arXiv:1208.4177, 2012.

[2] Byun, S., and L. WAng: Elliptic equations with BMO nonlinearity in Reifenberg domains. Adv. Math. 219:6, 2008, 1937-1971.

[3] Byun, S., and L. WANG: Gradient estimates for elliptic systems in non-smooth domains. Math. Ann. 341:3, 2008, 629-650.

[4] Byun, S., L. Wang, and S. Zhou: Nonlinear elliptic equations with BMO coefficients in Reifenberg domains. - J. Funct. Anal. 250:1, 2007, 167-196.

[5] Calderón, A. P.: Lebesgue spaces of differentiable functions and distributions. - Proc. Sympos. Pure Math. IV, 1961, 33-49.

[6] Christ, M.: The extension problem for certain function spaces involving fractional orders of differentiability. - Ark. Mat. 22:1-2, 1984, 63-81.

[7] ChuA, S.-K.: Extension theorems on weighted Sobolev spaces. - Indiana Univ. Math. J. 41:4, 1992, 1027-1076.

[8] ChuA, S.-K.: Some remarks on extension theorems for weighted Sobolev spaces. - Illinois J. Math. 38:1, 1994, 95-126.

[9] ChuA, S.-K.: Extension theorems on weighted Sobolev spaces and some applications. - Canad. J. Math. 58:3, 2006, 492-528.

[10] DAvid, G.: Hölder regularity of two-dimensional almost-minimal sets in $\mathbf{R}^{n}$. - Ann. Fac. Sci. Toulouse Math. (6) 18:1, 2009, 1, 65-246.

[11] DAvid, G.: $C^{1+\alpha}$-regularity for two dimensional almost-minimal sets in $\mathbf{R}^{n}$. - J. Geom. Anal. 20:4, 2010, 837-954.

[12] David, G.: Approximation of a Reifenberg-flat set by a smooth surface. - Bull. Belg. Math. Soc. Simon Stevin (to appear).

[13] David, G., T. De Pauw, and T. Toro: A generalization of Reifenberg's theorem in $\mathbf{R}^{3}$. Geom. Funct. Anal. 18:4, 2008, 1168-1235. 
[14] Davies, E. B.: Properties of the Green's functions of some Schrödinger operators. - J. London Math. Soc. (2) 7, 1974, 483-491.

[15] HajŁasz, P., and P. Koskela: Isoperimetric inequalities and imbedding theorems in irregular domains. - J. London Math. Soc. (2) 58:2, 1998, 425-450.

[16] Hong, G., and L. WAng: A geometric approach to the topological disk theorem of Reifenberg. - Pacific J. Math. 233:2, 2007, 321-339.

[17] Hong, G., and L. Wang: A new proof of Reifenberg's topological disc theorem. - Pacific J. Math. 246:2, 2010, 325-332.

[18] Jerison, D., and C. Kenig: Boundary behavior of harmonic functions in nontangentially accessible domains. - Adv. Math., 46:1, 1982, 80-147.

[19] Jones, P.: Quasiconformal mappings and extendability of functions in Sobolev spaces. - Acta Math. 147:1-2, 1981, 71-88.

[20] Kenig, C., and T. Toro: Harmonic measure on locally flat domains. - Duke Math. J. 87:3, $1997,509-551$.

[21] Kenig, C., and T. Toro: Free boundary regularity for harmonic measures and Poisson kernels. - Ann. of Math. (2) 150:2, 1999, 369-454.

[22] Kenig, C., and T. Toro: Poisson kernel characterization of Reifenberg flat chord arc domains. - Ann. Sci. Éc. Norm. Supér. (4) 36:3, 2003, 323-401.

[23] Lemenant, A.: Energy improvement for energy minimizing functions in the complement of generalized Reifenberg-flat sets. - Ann. Sc. Norm. Super. Pisa Cl. Sci. (5) 9, 2010, 1-34.

[24] Lemenant, A.: Regularity of the singular set for Mumford-Shah minimizers in $\mathbf{R}^{3}$ near a minimal cone. - Ann. Sc. Norm. Super. Pisa Cl. Sci. (5) 10:3, 2011, 561-609.

[25] Lemenant, A., and E. Milakis: Quantitative stability for the first Dirichlet eigenvalue in Reifenberg-flat domains in $\mathbf{R}^{n}$. - J. Math. Anal. Appl. 364, 2010, 522-533.

[26] Lemenant, A., and E. Milakis: A stability result for Nonlinear Neumann problems in Reifenberg flat domains in $\mathbf{R}^{n}$. - Publ. Mat. 55:2, 2011, 413-432.

[27] Lemenant, A., E. Milakis, and L. V. Spinolo: Spectral stability estimates for the Dirichlet and Neumann Laplacian in rough domains. - J. Funct. Anal. 264:9, 2013, 2097-2135.

[28] Milakis, E., and T. Toro: Divergence form operators in Reifenberg flat domains. - Math. Z. 264:1,2010, 15-41.

[29] Reifenberg, E. R.: Solution of the Plateau Problem for $m$-dimensional surfaces of varying topological type. - Acta Math. 104, 1960, 1-92.

[30] Ross, M.: The Lipschitz continuity of Neumann eigenvalues on convex domains. - Hokkaido Math. J. 33:2, 2004, 369-381.

[31] Stein, E. M.: Singular integrals and differentiability properties of functions. - Princeton Math. Ser., No. 30, Princeton Univ. Press, Princeton, N.J., 1970.

[32] Toro, T.: Doubling and flatness: geometry of measures. - Notices Amer. Math. Soc. 44:9, 1997, 1087-1094.

[33] Toro, T.: Geometry of measures: harmonic analysis meets geometric measure theory. - In: Handbook of geometric analysis, No. 1, Adv. Lect. Math. (ALM) 7, Int. Press, Somerville, MA, 2008, 449-465.

Received 12 September $2012 \bullet$ Accepted 17 May 2013 